\title{
UNIVERSALITY LIMITS IN THE BULK FOR ARBITRARY MEASURES ON COMPACT SETS
}

\author{
D. S. LUBINSKY
}

\begin{abstract}
We present a new method for establishing universality limits in the bulk, based on the theory of entire functions of exponential type. Let $\mu$ be a measure on a compact subset of the real line. Assume that $\mu$ is absolutely continuous in a neighborhood of some point $x$ in the support, and that $\mu^{\prime}$ is bounded above and below near $x$, which is assumed to be a Lebesgue point of $\mu^{\prime}$. Then universality holds at $x$ iff it holds "along the diagonal", that is

$$
\lim _{n \rightarrow \infty} \frac{K_{n}\left(x+\frac{a}{n}, x+\frac{a}{n}\right)}{K_{n}(x, x)}=1
$$

for all real $a$. The method does not require regularity of the measure $\mu$ as did earlier methods. Moreover, the assumption on the diagonal is certainly satisfied in the case of regular measures, so that we obtain another proof of some recent results of Simon and Totik.
\end{abstract}

\section{Introduction AND Results ${ }^{1}$}

Let $\mu$ be a finite positive Borel measure with compact support $\operatorname{supp}[\mu]$ and infinitely many points in the support. Then we may define orthonormal polynomials

$$
p_{n}(x)=\gamma_{n} x^{n}+\ldots, \gamma_{n}>0,
$$

$n=0,1,2, \ldots$ satisfying the orthonormality conditions

$$
\int p_{n} p_{m} d \mu=\delta_{m n}
$$

Throughout we use

$$
w=\frac{d \mu}{d x}
$$

to denote the Radon-Nikodym derivative of $\mu$. We say that $\mu$ is regular (in the sense of Stahl and Totik [23]) if

$$
\lim _{n \rightarrow \infty} \gamma_{n}^{1 / n}=\frac{1}{\operatorname{cap}(\operatorname{supp}[\mu])},
$$

where cap denotes logarithmic capacity. When the support is $[-1,1]$, the condition reduces to

$$
\lim _{n \rightarrow \infty} \gamma_{n}^{1 / n}=2
$$

Date: December 2, 2007.

${ }^{1}$ Research supported by NSF grant DMS0400446 and US-Israel BSF grant 2004353 
Orthogonal polynomials play an important role in random matrix theory $[1],[2],[8],[18]$. One of the key limits there involves the reproducing kernel

$$
K_{n}(x, y)=\sum_{k=0}^{n-1} p_{k}(x) p_{k}(y) .
$$

Because of the Christoffel-Darboux formula, it may also be expressed as

$$
K_{n}(x, y)=\frac{\gamma_{n-1}}{\gamma_{n}} \frac{p_{n}(x) p_{n-1}(y)-p_{n-1}(x) p_{n}(y)}{x-y} .
$$

Define the normalized kernel

$$
\widetilde{K}_{n}(x, y)=w(x)^{1 / 2} w(y)^{1 / 2} K_{n}(x, y) .
$$

The simplest case of the universality law is the limit

$$
\lim _{n \rightarrow \infty} \frac{\widetilde{K}_{n}\left(\xi+\frac{a}{\widetilde{K}_{n}(\xi, \xi)}, \xi+\frac{b}{\widetilde{K}_{n}(\xi, \xi)}\right)}{\widetilde{K}_{n}(\xi, \xi)}=\frac{\sin \pi(a-b)}{\pi(a-b)} .
$$

Typically this holds uniformly for $\xi$ in a compact subinterval of $(-1,1)$ and $a, b$ in compact subsets of the real line. Of course, when $a=b$, we interpret $\frac{\sin \pi(a-b)}{\pi(a-b)}$ as 1 .

In [15], we presented a new approach, based on localization and smoothing. It was assumed that $\mu$ is a finite positive measure with support $[-1,1]$, that is regular (in the sense of Stahl and Totik). Moreover, it was assumed that in a neighborhood of some compact set $J \subset(-1,1), \mu$ is absolutely continuous, while $w=\mu^{\prime}$ is positive and continuous at each point of $J$. The universality limit (1.4) was established uniformly for $\xi \in J$ and $a, b$ in compact subsets of the real line. If $J$ consists of just a single point $x$, then the hypothesis is that $\mu$ is absolutely continuous in some neighborhood $(x-\varepsilon, x+\varepsilon)$ of $x$, while $w(x)>0$ and $w$ is continuous at $x$. This alone is sufficient for universality at $x$.

Subsequently, Totik [26], his student Findley [3], and Simon [22] presented far reaching extensions of this result. For example, Totik showed that the same result holds for regular measures on a general compact subset of the real line, instead of $[-1,1]$, and moreover, we may relax the requirement of continuity of $w$. We only need $\log w$ to be integrable in a neighborhood of the points where universality is desired, together with a Lebesgue point type condition on a certain local Szegö function. In particular, we obtain universality a.e. in any neighborhood where $\log w$ is integrable. Totik's method was based on that in [15], together with "polynomial pullbacks" and potential theory. Simon [22] used the ideas of [15] together with Jost functions, and other tools, to prove universality at points of continuity for any regular measure. We emphasize that all these results require regularity of the measure $\mu$, and use this property in an essential way.

Traditionally, universality results had been proved by substituting higher order asymptotics into the Christoffel-Darboux formula. Levin [11] observed 
that mere first order asymptotics are sufficient. The error can then be controlled by a suitable Markov-Bernstein inequality. Together with the method of [15], Levin's fundamental idea lead to universality results for fixed exponential weights on the real line. This circle of methods has also been exploited for orthogonal polynomials on the unit circle, and for universality at the edge of the spectrum [12], [13], [16], [17].

In this paper, we present a new method, based on the theory of entire functions of exponential type, that works for arbitrary, possibly non-regular, measures with compact support. The hypotheses involve the kernel along the diagonal, or its reciprocal, the Christoffel function

$$
\lambda_{n}(x)=1 / K_{n}(x, x) .
$$

Our main result is that universality is equivalent to "universality along the diagonal", or alternatively, ratio asymptotics for Christoffel functions.

\section{Theorem 1.1}

Let $\mu$ be a finite positive Borel measure on the real line with compact support. Let $J \subset \operatorname{supp}[\mu]$ be compact, and such that $\mu$ is absolutely continuous in an open set containing J. Assume that $w$ is positive and continuous at each point of $J$. The following are equivalent:

(I) Uniformly for $\xi \in J$ and a in compact subsets of the real line,

$$
\lim _{n \rightarrow \infty} \frac{K_{n}\left(\xi+\frac{a}{n}, \xi+\frac{a}{n}\right)}{K_{n}(\xi, \xi)}=1 .
$$

(II) Uniformly for $\xi \in J$ and $a, b$ in compact subsets of the complex plane, we have

$$
\lim _{n \rightarrow \infty} \frac{K_{n}\left(\xi+\frac{a}{\widetilde{K}_{n}(\xi, \xi)}, \xi+\frac{b}{\widetilde{K}_{n}(\xi, \xi)}\right)}{K_{n}(\xi, \xi)}=\frac{\sin \pi(a-b)}{\pi(a-b)} .
$$

\section{Remarks}

(a) If we restrict $a, b$ to lie in compact subsets of the real line, then we may reformulate the limit (1.7) as

$$
\lim _{n \rightarrow \infty} \frac{\tilde{K}_{n}\left(\xi+\frac{a}{\widetilde{K}_{n}(\xi, \xi)}, \xi+\frac{b}{\widetilde{K}_{n}(\xi, \xi)}\right)}{\tilde{K}_{n}(\xi, \xi)}=\frac{\sin \pi(a-b)}{\pi(a-b)},
$$

since the quantity on the left is well defined.

(b) Of course the hypothesis (1.6) can be reformulated in terms of Christoffel functions: uniformly for $\xi \in J$ and $a$ in compact subsets of the real line,

$$
\lim _{n \rightarrow \infty} \lambda_{n}\left(\xi+\frac{a}{n}\right) / \lambda_{n}(\xi)=1 .
$$

If we assume instead of (1.6), that for some subsequence $\mathcal{S}$ of integers,

$$
\lim _{n \rightarrow \infty, n \in \mathcal{S}} \lambda_{n}\left(\xi+\frac{a}{n}\right) / \lambda_{n}(\xi)=1,
$$


again with the relevant uniformity, then the conclusion (1.7) holds as $n \rightarrow \infty$ through $\mathcal{S}$.

(c) We can reformulate the hypotheses so that we avoid assuming uniformity in $a$. Instead of (1.6), we can assume that for each fixed $a$, we have uniformly for $\xi \in J$,

$$
\lim _{n \rightarrow \infty} \frac{K_{n}\left(\xi+\frac{a}{\widetilde{K}_{n}(\xi, \xi)}, \xi+\frac{a}{\widetilde{K}_{n}(\xi, \xi)}\right)}{K_{n}(\xi, \xi)}=1 .
$$

Moreover, instead of assuming this for all $a$, it suffices that it holds for a denumerable set of $a$ with a finite limit point.

(d) If $J$ consists of a single point $\xi$, and we make the weaker hypothesis

$$
\liminf _{n \rightarrow \infty} \lambda_{n}\left(\xi+\frac{a}{n}\right) / \lambda_{n}(\xi) \geq 1
$$

then we can still prove (1.7) when $a=0$ or $b=0$. However for general $a, b$ we cannot prove this.

(e) If $\mu$ is a regular measure with support $E$, then it is known that for $\xi \in J$,

$$
\lim _{n \rightarrow \infty} n \lambda_{n}\left(\xi+\frac{a}{n}\right)=w(\xi) / \nu_{E}(\xi),
$$

where $\nu_{E}$ is the equilibrium measure of $E$. This remarkable result was proved by Totik [26], [25], extending a great deal of earlier work. Totik actually proved convergence a.e., but as noted in [26], his proof also establishes the limit uniformly in compact sets where there is continuity, allowing in addition the parameter $a$. Another proof is given in [22]. So for regular measures on general compact sets, we obtain an alternative, and more direct, proof of many of the aforementioned results of Totik [26] and Simon $[22]$.

(f) It is likely that the ratio limit (1.6) can hold even when

$$
\lim _{n \rightarrow \infty} \frac{1}{n} K_{n}(x, x)
$$

does not exist.

Instead of assuming continuity on $J$, we can assume a Lebesgue point type condition. It was Vili Totik who first observed that universality can be proved at Lebesgue points, rather than just points of continuity [26].

\section{Theorem 1.2}

Let $\mu$ be a finite positive Borel measure with compact support. Let $J \subset \operatorname{supp}[\mu]$ be compact, and such that $\mu$ is absolutely continuous in an open set containing J. Assume that $w$ is bounded above and below by positive constants in that open set. Assume, moreover, that uniformly for $\xi \in J$, we have

$$
\lim _{s \rightarrow 0+} \frac{1}{s} \int_{\xi-s}^{\xi+s}|w(t)-w(\xi)| d t=0 .
$$

Then the equivalence of (I), (II) in Theorem 1.1 remains valid. 
Of course, when $J=\{\xi\}$, (1.11) just asserts that $\xi$ is a Lebesgue point of $w$. When $J$ has non-empty interior, the uniformity of (1.11) forces $w$ to be continuous in the interior of $J$. As noted above, for regular measures, Totik and Findley assumed less on $w$. They assumed that $\log w$ is integrable in a neighborhood of $\xi$, rather than $w$ being bounded above and below by positive constants there. The methods of this paper may also be applied to varying weights [14].

The paper is organised as follows. In Section 2, we present the ideas of proof. In Section 3, we present further notation and background. In section 4, we survey some results that we shall need on entire functions of exponential type. In Section 5, we use the theory of normal families to establish some preliminary results. In Section 6, we prove Theorem 1.2, and deduce Theorem 1.1.

\section{The Ideas of Proof}

Suppose that $\mu$ is a measure with compact support and that $w=\mu^{\prime}$ is bounded above and below in some open set containing the compact set $J$. Then it is well known that for some $C_{1}, C_{2}>0$,

$$
C_{1} \leq \frac{1}{n} K_{n}(x, x) \leq C_{2}
$$

in any proper open subset of that open set, and hence, via Cauchy-Schwarz inequality, that

$$
\frac{1}{n}\left|K_{n}(\xi, t)\right| \leq C
$$

for $\xi, t$ in the latter open set. We can use this and Bernstein's inequality

$$
|P(z)| \leq\left|z+\sqrt{z^{2}-1}\right|^{n}\|P\|_{L_{\infty}[-1,1]}, z \in \mathbb{C},
$$

valid for polynomials $P$ of degree $\leq n$, to show that for fixed $\xi \in J$, and $a, b$ in any given compact subset of the complex plane,

$$
\left\{\frac{1}{n} K_{n}\left(\xi+\frac{a}{n}, \xi+\frac{b}{n}\right)\right\}_{n=1}^{\infty}
$$

is uniformly bounded. Hence it is a normal family. In view of (2.1), the same is true of $\left\{f_{n}(a, b)\right\}_{n=1}^{\infty}$, where

$$
f_{n}(a, b)=\frac{K_{n}\left(\xi+\frac{a}{\tilde{K}_{n}(\xi, \xi)}, \xi+\frac{b}{\tilde{K}_{n}(\xi, \xi)}\right)}{K_{n}(\xi, \xi)} .
$$

In fact, Bernstein's inequality yields a lot more: there exist $C_{1}, C_{2}>0$ with the following property. Given $A>0$, we have for $n \geq n_{0}(A)$ and $|a|,|b| \leq A$, that

$$
\left|f_{n}(a, b)\right| \leq C_{1} e^{C_{2}(|\operatorname{Im} a|+|\operatorname{Im} b|)} .
$$

We emphasize that $C_{1}$ and $C_{2}$ are independent of $n, A, a, b$. 
Let $f(a, b)$ be the limit of some subsequence $\left\{f_{n}(\cdot, \cdot)\right\}_{n \in \mathcal{S}}$ of $\left\{f_{n}(\cdot, \cdot)\right\}_{n=1}^{\infty}$. It is an entire function in $a, b$, but (2.3) shows even more: namely that for all complex $a, b$,

$$
|f(a, b)| \leq C_{1} e^{C_{2}(|\operatorname{Im} a|+|\operatorname{Im} b|)} .
$$

So $f$ is bounded for $a, b \in \mathbb{R}$, and is an entire function of exponential type in each variable. We can then apply the very rich theory of entire functions of exponential type.

Our goal is to show that

$$
f(a, b)=\frac{\sin \pi(a-b)}{\pi(a-b)} .
$$

So we study the properties of $f$. Our main tool is to take elementary properties of the reproducing kernel $K_{n}$, and then after scaling and taking limits, to deduce that an analogous property is true for $f$. Let us fix $a$. Since for each real $\xi, K_{n}(\xi, t)$ has only real zeros, the same is true of $f(a, \cdot)$. Moreover, $f(a, \cdot)$ has countably many such zeros. Using elementary properties of the reproducing kernel $K_{n}$, we can show that for all $a \in \mathbb{C}$,

$$
\int_{-\infty}^{\infty}|f(a, s)|^{2} d s \leq f(a, \bar{a}) .
$$

If $\sigma$ is the exponential type of $f(a, \cdot)$, we can show that $\sigma$ is independent of $a$, using interlacing properties of zeros of $K_{n}$. Using the fact that $\frac{\sin \pi s}{\pi s}$ is a reproducing kernel for the entire functions of exponential type that are also in $L_{2}(\mathbb{R})$, we can establish the useful inequality

$$
\begin{aligned}
& 0 \leq \int_{\mathbb{R}}\left(\frac{f(a, s)}{f(a, a)}-\frac{\sin \sigma(s-a)}{\sigma(s-a)}\right)^{2} d s \\
\leq & \frac{1}{f(a, a)}-\frac{\pi}{\sigma} .
\end{aligned}
$$

From this we deduce

$$
\sigma \geq \pi \sup _{x \in \mathbb{R}} f(x, x) \geq \pi
$$

For the converse inequality, we use Markov-Stieltjes inequalities, and a formula relating exponential type of entire functions and their zero distribution, to obtain

$$
\sigma \leq \pi \sup _{x \in \mathbb{R}} f(x, x) .
$$

Thus,

$$
\sigma=\pi \sup _{x \in \mathbb{R}} f(x, x),
$$

and (2.7) becomes

$$
\begin{aligned}
& \int_{-\infty}^{\infty}\left(\frac{f(a, s)}{f(a, a)}-\frac{\sin \sigma(a-s)}{\sigma(a-s)}\right)^{2} d s \\
\leq & \frac{1}{f(a, a)}-\frac{1}{\sup _{x \in \mathbb{R}} f(x, x)} .
\end{aligned}
$$


Assuming (1.6), we immediately obtain $f(x, x)=1$ for all $x$, and then $\sigma=\pi$. Substituting this back into (2.8), completes the proof of Theorem 1.2 .

\section{Notation AND BACKGROUND}

In this section, we record our notation, though some of it has already been introduced earlier. In the sequel $C, C_{1}, C_{2}, \ldots$ denote constants independent of $n, x, y, s, t$. The same symbol does not necessarily denote the same constant in different occurences. We shall write $C=C(\alpha)$ or $C \neq C(\alpha)$ to respectively denote dependence on, or independence of, the parameter $\alpha$. We use $\sim$ in the following sense: given real sequences $\left\{c_{n}\right\},\left\{d_{n}\right\}$, we write

$$
c_{n} \sim d_{n}
$$

if there exist positive constants $C_{1}, C_{2}$ with

$$
C_{1} \leq c_{n} / d_{n} \leq C_{2} \text {. }
$$

Similar notation is used for functions and sequences of functions.

Throughout, $\mu$ denotes a finite positive Borel measure with compact support. Its Radon-Nikodym derivative is $w=\mu^{\prime}$. The corresponding orthonormal polynomials are denoted by $\left\{p_{n}\right\}_{n=0}^{\infty}$, so that

$$
\int p_{n} p_{m} d \mu=\delta_{m n}
$$

We denote the zeros of $p_{n}$ by

$$
x_{n n}<x_{n-1, n}<\ldots<x_{2 n}<x_{1 n} .
$$

The reproducing kernel $K_{n}(x, t)$, is defined by (1.2), while the normalized reproducing kernel is defined by (1.3). The $n$th Christoffel function is

$$
\lambda_{n}(x)=1 / K_{n}(x, x)=\inf _{\operatorname{deg}(P) \leq n-1} \frac{\int P^{2} d \mu}{P^{2}(x)} .
$$

The Gauss quadrature formula asserts that whenever $P$ is a polynomial of degree $\leq 2 n-1$,

$$
\sum_{j=1}^{n} \lambda_{n}\left(x_{j n}\right) P\left(x_{j n}\right)=\int P d \mu .
$$

In addition to this, we shall need another Gauss type of quadrature formula [4, p. $19 \mathrm{ff}$.$] . Given a real number \xi$, there are $n$ or $n-1$ points $t_{j n}=t_{j n}(\xi)$, one of which is $\xi$, such that

$$
\sum_{j} \lambda_{n}\left(t_{j n}\right) P\left(t_{j n}\right)=\int P d \mu,
$$

whenever $P$ is a polynomial of degree $\leq 2 n-2$. The $\left\{t_{j n}\right\}$ are zeros of

$$
\psi_{n}(\xi, t)=p_{n}(\xi) p_{n-1}(t)-p_{n-1}(\xi) p_{n}(t),
$$

regarded as a function of $t$. 
In order to prove that universality holds uniformly for $\xi$ in $J$, we shall consider a sequence $\left\{\xi_{n}\right\}$ of points in $J$, rather than a fixed $\xi$. At the $n$th stage, we shall consider the quadrature that includes $\xi_{n}$, so that

$$
t_{j n}=t_{j n}\left(\xi_{n}\right) \text { for all } j .
$$

Because we wish to focus on $\xi_{n}$, we shall set $t_{0 n}=\xi_{n}$, and order the $\left\{t_{j n}\right\}$ around $\xi_{n}$, treated as the origin:

$$
\ldots<t_{-2, n}<t_{-1, n}<t_{0 n}=\xi_{n}<t_{1 n}<\ldots .
$$

Of course the sequence $\left\{t_{j n}\right\}$ consists of either $n-1$ or $n$ points, so terminates, and it is possible that all $t_{j n}$ lie to the left or right of $\xi_{n}$. However in the limiting situations we treat, where $\xi_{n}$ lies in the interior of the support, this will not occur. It is known $\left[4\right.$, p. 19] that when $\left(p_{n} p_{n-1}\right)\left(\xi_{n}\right) \neq 0$, then one zero of $\psi_{n}\left(\xi_{n}, t\right)$ lies in $\left(x_{j n}, x_{j-1, n}\right)$ for each $j$, and the remaining zero lies outside $\left(x_{n n}, x_{1 n}\right)$.

Throughout $J$ will be the compact set in Theorem 1.2. We let $J_{1}$ and $J_{2}$ denote compact sets, each consisting of finitely many closed intervals, such that

$$
J_{2}^{o} \supset J_{1} \text { and } J_{1}^{o} \supset J
$$

while $\mu$ is absolutely continuous in $J_{2}$, with

$$
C_{1} \leq w \leq C_{2} \text { in } J_{2} .
$$

Recall, we assumed that $w$ is bounded above and below in an open set containing $J$, so such a $J_{1}$ and $J_{2}$ exist. Furthermore, since $J_{1}$ and $J_{2}$ consist of finitely many intervals, we may assume they are each just one interval. For, proving Theorem in 1.2 uniformly for that part of $J$ contained in such intervals, then yields the uniformity in the general case. So in the sequel, we assume $J_{1}$ and $J_{2}$ are compact intervals.

For the given sequence $\left\{\xi_{n}\right\}$ in $J$, we shall define for $n \geq 1$,

$$
f_{n}(a, b)=\frac{K_{n}\left(\xi_{n}+\frac{a}{\tilde{K}_{n}\left(\xi_{n}, \xi_{n}\right)}, \xi_{n}+\frac{b}{\tilde{K}_{n}\left(\xi_{n}, \xi_{n}\right)}\right)}{K_{n}\left(\xi_{n}, \xi_{n}\right)} .
$$

The zeros of

$$
f_{n}(0, t)=\frac{K_{n}\left(\xi_{n}, \xi_{n}+\frac{t}{\tilde{K}_{n}\left(\xi_{n}, \xi_{n}\right)}\right)}{K_{n}\left(\xi_{n}, \xi_{n}\right)}
$$

will be denote by $\left\{\rho_{j n}\right\}_{j \neq 0}$. Since $\left\{t_{j n}\right\}=\left\{t_{j n}\left(\xi_{n}\right)\right\}$ are the zeros of $\psi_{n}\left(\xi_{n}, t\right)=\left(\frac{\gamma_{n-1}}{\gamma_{n}}\right)^{-1}\left(\xi_{n}-t\right) K_{n}\left(\xi_{n}, t\right)$, we have

$$
\rho_{j n}=\tilde{K}_{n}\left(\xi_{n}, \xi_{n}\right)\left(t_{j n}-\xi_{n}\right) .
$$

We also set,

$$
\rho_{0 n}=0,
$$

corresponding to $t_{0 n}=\xi_{n}$. 
For an appropriate subsequence $\mathcal{S}$ of integers, we shall let

$$
f(a, b)=\lim _{n \rightarrow \infty, n \in \mathcal{S}} f_{n}(a, b) .
$$

The zeros of $f(0, \cdot)$ will be denoted by $\left\{\rho_{j}\right\}_{j \neq 0}$, and we set $\rho_{0}=0$. Our ordering of zeros is

$$
\cdots \leq \rho_{-2} \leq \rho_{-1}<\rho_{0}=0<\rho_{1} \leq \rho_{2} \leq \ldots .
$$

We shall denote the (exponential) type of $f(a, \cdot)$ by $\sigma_{a}$. We shall show that $\sigma_{a}$ is independent of $a$, and then just use $\sigma$ to denote the type. Initially, this type will be associated with the specific subsequence $\mathcal{S}$.

\section{Entire functions of Exponential Type}

In this section, we review some theory that we shall use about entire functions of exponential type. Most of this can be found in the elegant series of lectures of B. Ja. Levin [10]. Recall that if $g$ is entire of order 1, then its exponential type $\sigma$ is

$$
\sigma=\limsup _{r \rightarrow \infty} \frac{\max _{|z|=r} \log |g(z)|}{r} .
$$

We say that an entire function $g$ belongs to the Cartwright class and write $g \in \mathcal{C}$ if it is of exponential type and

$$
\int_{-\infty}^{\infty} \frac{\log ^{+}|g(t)|}{1+t^{2}} d t<\infty
$$

Here $\log ^{+} s=\max \{0, \log s\}$.

We let $n(g, r)$ denote the number of zeros of $g$ in the ball center 0 , radius $r$, counting multiplicity. An important result is that for $g \in \mathcal{C}$, that is real valued on the real axis,

$$
\lim _{r \rightarrow \infty} \frac{n(g, r)}{2 r}=\frac{\sigma}{\pi}
$$

For this, see [10, Theorem 1, p. 127]. A simpler proof, in the case of $g$ having all real zeros, is given in $[7, \mathrm{p} .66]$.

When $f$ is entire of exponential type $\sigma$ and bounded along the real axis, we have $[10$, p. 38 , Theorem 3$]$

$$
|f(z)| \leq e^{\sigma|\operatorname{Im} z|}\|f\|_{L_{\infty}(\mathbb{R})}, z \in \mathbb{C} .
$$

When $g$ is entire of exponential type $\sigma$ and $g \in L_{2}(\mathbb{R})$, we write, as did Levin, $g \in L_{\sigma}^{2}$. Here, we have instead of the last inequality, [10, p. 149]

$$
|g(z)| \leq\left(\frac{2}{\pi}\right)^{1 / 2} e^{\sigma(|\operatorname{Im} z|+1)}\|g\|_{L_{2}(\mathbb{R})}, z \in \mathbb{C} .
$$


An important identity is the reproducing kernel identity [24, p. 95], [6, (6.75), p. 58]

$$
g(x)=\int_{-\infty}^{\infty} g(t) \frac{\sin \sigma(x-t)}{\pi(x-t)} d t, x \in \mathbb{R}
$$

when $g \in L_{\sigma}^{2}$. One possible proof of this involves the Paley-Wiener Theorem, and the observation that $\frac{\sin \sigma(x-t)}{\pi(x-t)}$ is the Fourier transform of the characteristic function of $[-\sigma, \sigma]$. We shall also use [5, p. 414, no. 3.741.3] (or, choose $g(t)=\frac{\sin \pi(x-t)}{\pi(x-t)}$ in the previous identity)

$$
\int_{-\infty}^{\infty}\left(\frac{\sin \pi s}{\pi s}\right)^{2} d s=1
$$

\section{NormaLity}

We begin with a straightforward consequence of Bernstein's growth inequality for polynomials in the complex plane. Throughout this section, $J, J_{1}$ and $J_{2}$ are as in Section 3, so that $J_{1}$ and $J_{2}$ are closed intervals, while $\left\{\xi_{n}\right\}$ is a sequence in $J$. We shall assume the hypotheses of Section 3, but shall not assume (1.6).

\section{Lemma 5.1}

Let $[c, d]$ be a real interval and $\mathcal{K}$ be a compact subset of $(c, d)$. Let $A, \eta>0$ and

$$
\Gamma=\sup _{x \in \mathcal{K}} \frac{1}{\sqrt{(d-x)(x-c)}}
$$

There exists $n_{0}=n_{0}(A, \mathcal{K}, \eta, \Gamma)$ such that for $n \geq n_{0}$, polynomials $P$ of degree $\leq n, x \in \mathcal{K}$ and $|a| \leq A$,

$$
\left|P\left(x+i \frac{a}{n}\right)\right| \leq e^{(1+\eta) \Gamma|a|}\|P\|_{L_{\infty}[c, d]} .
$$

\section{Proof}

We prove this for $[c, d]=[-1,1]$. The general case follows by a linear transformation. Let $x \in \mathcal{K}$ and $z=x+i \frac{a}{n}$. By Bernstein's inequality,

$$
|P(z)| \leq\left|z+\sqrt{z^{2}-1}\right|^{n}\|P\|_{L_{\infty}[-1,1]} .
$$


As $\left|x+\sqrt{x^{2}-1}\right|=1$, we obtain

$$
\begin{aligned}
& \log \left|z+\sqrt{z^{2}-1}\right| \\
= & \operatorname{Re}\left(\int_{0}^{a / n} \frac{d}{d u} \log \left(u+\sqrt{u^{2}-1}\right)_{\mid u=x+i s} i d s\right) \\
= & \operatorname{Re}\left(\int_{0}^{a / n} \frac{1}{\sqrt{u^{2}-1}}{ }_{\mid u=x+i s} i d s\right) \\
= & \frac{1}{\sqrt{1-x^{2}}} \operatorname{Re}\left(\int_{0}^{a / n} \frac{d s}{\sqrt{1+\frac{s^{2}-2 i x s}{1-x^{2}}}}\right) \\
= & \frac{1}{\sqrt{1-x^{2}}}\left(\frac{a}{n}+O\left(\frac{a}{n}\right)^{2} /\left(1-x^{2}\right)\right) .
\end{aligned}
$$

On substituting this into (5.2), we obtain (5.1) in the special case $[c, d]=$ $[-1,1]$.

Lemma 5.2

For $n \geq 1$, let

$$
f_{n}(u, v)=\frac{K_{n}\left(\xi_{n}+\frac{u}{\tilde{K}_{n}\left(\xi_{n}, \xi_{n}\right)}, \xi_{n}+\frac{v}{\tilde{K}_{n}\left(\xi_{n}, \xi_{n}\right)}\right)}{K_{n}\left(\xi_{n}, \xi_{n}\right)}
$$

(a) $\left\{f_{n}(u, v)\right\}_{n=1}^{\infty}$ is uniformly bounded for $u, v$ in compact subsets of the plane.

(b) Let $f(u, v)$ denote the locally uniform limit of some subsequence $\left\{f_{n}(u, v)\right\}_{n \in \mathcal{S}}$ of $\left\{f_{n}(u, v)\right\}_{n=1}^{\infty}$. Then for each fixed real number $u, f(u, \cdot)$ is an entire function of exponential type. Moreover, for some $C_{1}$ and $C_{2}$ independent of $u, v$, and the subsequence $\mathcal{S}$,

$$
|f(u, v)| \leq C_{1} e^{C_{2}(|\operatorname{Im} u|+|\operatorname{Im} v|)} .
$$

(c) For each fixed real number $u, f(u, \cdot)$ has only real zeros.

\section{Proof}

(a) We note first that since $\mu$ is absolutely continuous in the larger interval $J_{2}$, and $w$ is bounded above and below there (recall (3.7)), we have the well known bound [19, Theorem 20, p. 116]

$$
K_{n}(x, x)^{-1}=\lambda_{n}(x) \sim \frac{1}{n} \text { in } J_{1} .
$$


Indeed, in one direction, this follows by comparing $\lambda_{n}(x)$ to the Christoffel functions of weights that are constant in $J_{2}$. By Cauchy-Schwarz, we have

$$
\begin{aligned}
& \frac{1}{n}\left|K_{n}(\xi, t)\right| \\
\leq & \left(\frac{1}{n} K_{n}(\xi, \xi)\right)^{1 / 2}\left(\frac{1}{n} K_{n}(t, t)\right)^{1 / 2} \leq C
\end{aligned}
$$

for $\xi, t \in J_{1}$. By Lemma 5.1, applied separately in each variable, we then have for $\xi, t \in J_{1},|a|,|b| \leq A$ and $n \geq n_{0}(A)$,

$$
\frac{1}{n}\left|K_{n}\left(\xi+i \frac{a}{n}, t+i \frac{b}{n}\right)\right| \leq C e^{C_{2}(|a|+|b|)} .
$$

(Strictly speaking, we have to take a slightly smaller interval than $J_{1}$, but can relabel). $C_{2}$ is independent of $A, \xi, t, a, b$. It depends only on the intervals $J_{1}$ and $J_{2}$. As $C$ is also independent of $A, \xi, a$, the stated uniform boundedness follows. Of course if $u, v$ lie in a bounded subset of the plane, and $\xi \in J_{1}$, then for $n$ large enough, we may write $\xi+\frac{u}{n}=\xi+\frac{\operatorname{Re}(u)}{n}+i \frac{\operatorname{Im}(u)}{n}$, where $\xi+\frac{\operatorname{Re}(u)}{n}$ is contained in a slightly large interval than $J_{1}$. By relabelling, we may assume it lies in $J_{1}$. Then we may recast (5.6) in the form

$$
\frac{1}{n}\left|K_{n}\left(\xi+\frac{u}{n}, \xi+\frac{v}{n}\right)\right| \leq C e^{C_{2}(|\operatorname{Im} u|+|\operatorname{Im} v|)} .
$$

Since

$$
\tilde{K}_{n}\left(\xi_{n}, \xi_{n}\right) \sim n
$$

we see also that for $|u|,|v| \leq A$ and $n \geq n_{0}(A)$

$$
\left|f_{n}(u, v)\right| \leq C e^{C_{2}(|\operatorname{Im} u|+|\operatorname{Im} v|)},
$$

where $C_{1}, C_{2}$ are independent of $n, u, v, A$.

(b) Now $\left\{f_{n}(u, v)\right\}_{n=1}^{\infty}$ is a normal family of two variables $u, v$. If $f(u, v)$ is the locally uniform limit through the subsequence $\mathcal{S}$ of integers, we see that $f(u, v)$ is an entire function in $u, v$ satisfying for all complex $u, v$,

$$
|f(u, v)| \leq C e^{C_{2}(|\operatorname{Im} u|+|\operatorname{Im} v|)} .
$$

In particular, $f(u, v)$ is bounded for $u, v \in \mathbb{R}$, and is an entire function of exponential type in each variable.

(c) It is shown in [4, p. 18], that for each real $\xi, K_{n}(\xi, t)$ has only real simple zeros. Hence for real $u, f_{n}(u, v)$ has only real simple zeros as a function of $v$. Hurwitz's theorem shows that $f(u, v)$ has only real zeros.

\section{Remark}

The only places in this paper where we use the upper bound $w \leq C$ are in deducing (5.5) above, and in Lemma 5.3(a) below. We can instead assume that there exists $C>0$, with the following property: given $A>0$, there exists $n_{0}=n_{0}(A)$ such that for $n \geq n_{0}, \xi \in J$, and $|a| \leq A$,

$$
K_{n}\left(\xi+\frac{a}{n}, \xi+\frac{a}{n}\right) \geq C n \text {. }
$$




\section{Lemma 5.3}

(a) Uniformly for $u \in \mathbb{R}$,

$$
f(u, u) \sim 1
$$

(b) Assume

$$
\lim _{\varepsilon \rightarrow 0+} \frac{1}{2 \varepsilon} \int_{\xi_{n}-\varepsilon}^{\xi_{n}+\varepsilon}\left|w(t)-w\left(\xi_{n}\right)\right| d t=0 .
$$

Then for all $u \in \mathbb{C}$,

$$
\int_{-\infty}^{\infty}|f(u, s)|^{2} d s \leq f(u, \bar{u}) .
$$

(c) For each $a \in \mathbb{R}, f(a, \cdot)$ has infinitely many real zeros.

\section{Proof}

(a) Since for some $\eta>0$, for $|t| \leq \eta$, and for large enough $n$,

$$
C_{1} \leq \frac{K_{n}\left(\xi_{n}+t, \xi_{n}+t\right)}{K_{n}\left(\xi_{n}, \xi_{n}\right)} \leq C_{2}
$$

(recall (5.5)), while

$$
f(u, u)=\lim _{n \rightarrow \infty, n \in \mathcal{S}} \frac{K_{n}\left(\xi_{n}+\frac{u}{\tilde{K}_{n}\left(\xi_{n}, \xi_{n}\right)}, \xi_{n}+\frac{u}{\tilde{K}_{n}\left(\xi_{n}, \xi_{n}\right)}\right)}{K_{n}\left(\xi_{n}, \xi_{n}\right)},
$$

we obtain (5.9).

(b) Let

$$
s=\xi_{n}+\frac{u}{\tilde{K}_{n}\left(\xi_{n}, \xi_{n}\right)} .
$$

We use the identity

$$
K_{n}(s, \bar{s})=\int\left|K_{n}(s, t)\right|^{2} d \mu(t),
$$

valid for all complex $s$. Let $r>0$. We drop most of the integral:

$$
\begin{aligned}
1 & \geq \int_{\xi_{n}-\frac{r}{\bar{K}_{n}\left(\xi_{n}, \xi_{n}\right)}}^{\xi_{n}+\frac{r}{\tilde{K}_{n}\left(\xi_{n}, \xi_{n}\right)}} \frac{\left|K_{n}(s, t)\right|^{2}}{K_{n}(s, \bar{s})} w(t) d t \\
& =w\left(\xi_{n}\right) \int_{\xi_{n}-\frac{r}{\bar{K}_{n}\left(\xi_{n}, \xi_{n}\right)}}^{\xi_{n}+\frac{r}{\tilde{K}_{n}\left(\xi_{n}, \xi_{n}\right)}} \frac{\left|K_{n}(s, t)\right|^{2}}{K_{n}(s, \bar{s})} d t+\int_{\xi_{n}-\frac{r}{\tilde{K}_{n}\left(\xi_{n}, \xi_{n}\right)}}^{\xi_{n}+\frac{r}{\tilde{K}_{n}\left(\xi_{n}, \xi_{n}\right)}} \frac{\left|K_{n}(s, t)\right|^{2}}{K_{n}(s, \bar{s})}\left(w(t)-w\left(\xi_{n}\right)\right) d t \\
& =: I_{1}+I_{2} .
\end{aligned}
$$

Here by Cauchy-Schwarz and the upper bound (5.6),

$$
\frac{\left|K_{n}(s, t)\right|^{2}}{K_{n}(s, \bar{s})} \leq K_{n}(t, t) \leq C n .
$$


Then by the lower bound implicit in (5.5),

$$
\begin{aligned}
\left|I_{2}\right| & \leq C n \int_{\xi_{n}-\frac{r}{\tilde{K}_{n}\left(\xi_{n}, \xi_{n}\right)}}^{\xi_{n}+\frac{r}{\bar{K}_{n}\left(\xi_{n}, \xi_{n}\right)}}\left|w(t)-w\left(\xi_{n}\right)\right| d t \\
& \leq \frac{C \tilde{K}_{n}\left(\xi_{n}, \xi_{n}\right)}{r} \int_{\xi_{n}-\frac{r}{\bar{K}_{n}\left(\xi_{n}, \xi_{n}\right)}}^{\xi_{n}+\frac{r}{\bar{K}_{n}\left(\xi_{n}, \xi_{n}\right)}}\left|w(t)-w\left(\xi_{n}\right)\right| d t \\
& \rightarrow 0, n \rightarrow \infty,
\end{aligned}
$$

by (5.10). Next, the substitution $t=\xi_{n}+\frac{y}{\tilde{K}_{n}\left(\xi_{n}, \xi_{n}\right)}$ and (5.12) give

$$
=\int_{-r}^{r}\left|\frac{K_{n}\left(\xi_{n}+\frac{u}{\tilde{K}_{n}\left(\xi_{n}, \xi_{n}\right)}, \xi_{n}+\frac{y}{K_{n}\left(\xi_{n}, \xi_{n}\right)}\right)}{\tilde{K}_{n}\left(\xi_{n}, \xi_{n}\right)}\right|^{2} \frac{K_{n}\left(\xi_{n}, \xi_{n}\right)}{K_{n}(s, \bar{s})} d y .
$$

As $n \rightarrow \infty$ through $\mathcal{S}$, the last right-hand side has lim inf at least

$$
\int_{-r}^{r} \frac{|f(u, y)|^{2}}{f(u, \bar{u})} d y
$$

Substituting into (5.13) gives

$$
1 \geq \int_{-r}^{r} \frac{|f(u, y)|^{2}}{f(u, \bar{u})} d y
$$

Finally, let $r \rightarrow \infty$.

(c) We note first that $f(a, \cdot)$ is non-constant, and not a polynomial. Indeed, we have just shown that it belongs to $L_{2}(\mathbb{R})$ and satisfies $f(a, a) \neq 0$. It also lies in the Cartwright class and is real on the real axis. We can then write $[10$, p. 130]

$$
f(a, a+z)=f(a, a) \lim _{R \rightarrow \infty} \prod_{b:|b|<R \text { and } f(a, a+b)=0}\left(1-\frac{z}{b}\right) .
$$

\section{Proof of Theorems 1.1 And 1.2}

It follows from Lemma 5.2 that for each real $a, f(a, \cdot)$ is entire of exponential type $\sigma_{a}$, say. We first show that $\sigma_{a}$ is independent of $a$. We note that $\sigma_{a}$ could possibly depend on $\left\{\xi_{n}\right\}$ and the subsequence $\mathcal{S}$.

\section{Lemma 6.1}

For $a \in \mathbb{R}$, let $n(f(a, \cdot), r)$ denote the the number of zeros of $f(a, \cdot)$ in the ball center 0 , radius $r$, counting multiplicity. Then for any real a, we have as $r \rightarrow \infty$,

$$
n(f(a, \cdot), r)-n(f(0, \cdot), r)=O(1)
$$


Consequently,

$$
\sigma_{a}=\sigma_{0}=\sigma, \text { say }
$$

Moreover, for all $a \in \mathbb{R}, f(a, \cdot) \in L_{\sigma}^{2}$.

\section{Proof}

We use a basic property of

$$
\psi_{n}(\xi, t)=\left(\frac{\gamma_{n-1}}{\gamma_{n}}\right)^{-1} K_{n}(\xi, t)(\xi-t)=p_{n}(t) p_{n-1}(\xi)-p_{n-1}(t) p_{n}(\xi)
$$

For real $\xi$, with $p_{n-1}(\xi) p_{n}(\xi) \neq 0, \psi_{n}(\xi, t)$ has, as a function of $t$, simple zeros in each of the intervals

$$
\left(x_{n n}, x_{n-1, n}\right),\left(x_{n-1, n}, x_{n-2, n}\right), \ldots,\left(x_{2 n}, x_{1 n}\right) .
$$

There is a single remaining zero, and this lies outside $\left[x_{n n}, x_{1 n}\right]$. When $p_{n-1}(\xi) p_{n}(\xi)=0, \psi_{n}(\xi, t)$ is a multiple of $p_{n}$ or $p_{n-1}$. As the zeros of the latter polynomials interlace, we see that in this case, there is a simple zero in each of the intervals

$$
\left[x_{n n}, x_{n-1, n}\right),\left[x_{n-1, n}, x_{n-2, n}\right), \ldots,\left[x_{2 n}, x_{1 n}\right) .
$$

For all this, see [4, proof of Theorem 3.1, p. 19]. It follows that whatever is $\xi$, the number $j$ of zeros of $K_{n}(\xi, t)$ in $\left[x_{m n}, x_{k n}\right]$ satisfies

$$
|j-(m-k)| \leq 1 \text {. }
$$

Consider now

$$
f_{n}(a, t)=K_{n}\left(\xi_{n}+\frac{a}{\tilde{K}_{n}\left(\xi_{n}, \xi_{n}\right)}, \xi_{n}+\frac{t}{\tilde{K}_{n}\left(\xi_{n}, \xi_{n}\right)}\right) / K_{n}\left(\xi_{n}, \xi_{n}\right)
$$

and

$$
f_{n}(0, t)=K_{n}\left(\xi_{n}, \xi_{n}+\frac{t}{\tilde{K}_{n}\left(\xi_{n}, \xi_{n}\right)}\right) / K_{n}\left(\xi_{n}, \xi_{n}\right)
$$

as functions of $t$. In any fixed interval $[-r, r]$, it follows that the difference between the number of zeros of these two functions is at most 2. Letting $n \rightarrow \infty$ through $\mathcal{S}$, we see that (6.1) holds. Then (6.2) follows from (4.3). Finally, $f \in L_{2}(\mathbb{R})$, by $(5.11)$, so also $f(a, \cdot) \in L_{\sigma}^{2}$.

\section{Lemma 6.2}

(a) Assume (5.10). Then we have for all $a \in \mathbb{R}$,

$$
\begin{aligned}
& \int_{-\infty}^{\infty}\left(\frac{f(a, s)}{f(a, a)}-\frac{\sin \sigma(a-s)}{\sigma(a-s)}\right)^{2} d s \\
\leq & \frac{1}{f(a, a)}-\frac{\pi}{\sigma} .
\end{aligned}
$$

$$
\sigma \geq \pi \sup _{a \in \mathbb{R}} f(a, a) \geq \pi
$$




\section{Proof}

(a) The left-hand side in (6.3) equals

$$
\begin{aligned}
& \frac{1}{f(a, a)^{2}} \int_{-\infty}^{\infty} f(a, s)^{2} d s-\frac{2}{f(a, a)} \int_{-\infty}^{\infty} f(a, s) \frac{\sin \sigma(a-s)}{\sigma(a-s)} d s+\int_{-\infty}^{\infty}\left(\frac{\sin \sigma(a-s)}{\sigma(a-s)}\right)^{2} d s \\
\leq & \frac{1}{f(a, a)}-2 \frac{\pi}{\sigma}+\frac{\pi}{\sigma},
\end{aligned}
$$

by (5.11) of Lemma 5.3(b), and the identities (4.6) and (4.7). Recall that $f(a, \cdot) \in L_{\sigma}^{2}$, so (4.6) is applicable.

(c) Since the left-hand side of (6.3) is nonnegative, we obtain for all real $a$,

$$
\sigma \geq \pi f(a, a) .
$$

As $f(0,0)=1$, we then obtain (6.4).

Recall from Section 3, the Gauss type quadrature formula, with nodes $\left\{t_{j n}\right\}$ including the point $\xi=\xi_{n}$ :

$$
\sum_{j} \lambda_{n}\left(t_{j n}\right) P\left(t_{j n}\right)=\int P(t) d \mu(t),
$$

for all polynomials $P$ of degree $\leq 2 n-2$. Recall that we order the nodes as

$$
\ldots<t_{-2, n}<t_{-1, n}<t_{0, n}=\xi_{n}<t_{1, n}<t_{2, n}<\ldots<.
$$

and write

$$
t_{j n}=\xi_{n}+\frac{\rho_{j n}}{\tilde{K}_{n}\left(\xi_{n}, \xi_{n}\right)} .
$$

\section{Lemma 6.3}

(a) For each fixed $j$, as $n \rightarrow \infty$ through $\mathcal{S}$,

$$
\rho_{j n} \rightarrow \rho_{j},
$$

where $\rho_{0}=0$ and

$$
\ldots \leq \rho_{-2} \leq \rho_{-1}<0<\rho_{1} \leq \rho_{2} \leq \ldots .
$$

(b) There exists $C_{1}$ such that for all integers $j$,

$$
\rho_{j}-\rho_{j-1} \leq C_{1} .
$$

(c) The function $f(0, z)$ has (possibly multiple) zeros at $\rho_{j}, j \neq 0$, and no other zeros.

Proof

(a), (c) We know that $f_{n}(0, z)=K_{n}\left(\xi_{n}, \xi_{n}+\frac{z}{\tilde{K}_{n}\left(\xi_{n}, \xi_{n}\right)}\right) / K\left(\xi_{n}, \xi_{n}\right)$ has simple zeros at $\rho_{j n}$, and no other zeros. Moreover as $n \rightarrow \infty$ through our subsequence, this sequence converges to $f(0, z)$, uniformly for $z$ in compact sets, and $f(0, z)$ is not identically 0 . The result then follows by Hurwitz' 
theorem.

(b) Fix $j$. We use the Markov-Stieltjes inequalities [4, (5.10), p. 33]:

$$
\int_{t_{j-1, n}}^{t_{j, n}} w \leq \int_{t_{j-1, n}}^{t_{j n}} d \mu \leq \lambda_{n}\left(t_{j-1, n}\right)+\lambda_{n}\left(t_{j n}\right) .
$$

Using our upper bounds for the Christoffel function, valid in an open set containing $J$, and the fact that each point of $J$ is a limit point of zeros [4, Theorem 2.4, p. 67], we see that the last right-hand side is $O\left(\frac{1}{n}\right)$. Moreover, by hypothesis, $w$ is bounded below in an open set containing $J$. It follows that for $n \geq n_{0}(j)$,

$$
t_{j n}-t_{j-1, n} \leq \frac{C}{n}
$$

It is crucial here that $C$ does nor depend on $j$, as it arises from the upper bound for the Christoffel functions and the lower bound for $w$. Then from (6.5) and (5.5), for $n \geq n_{0}(j)$,

$$
\rho_{j, n}-\rho_{j-1, n} \leq C,
$$

where $C$ is independent of $j$. Letting $n \rightarrow \infty$ through $\mathcal{S}$ gives the result.

We note that methods and results of Nevai [19, Theorem 20, p. 164] and Last and Simon [9, Sections 8 and 9] show more, namely that

$$
\frac{C_{1}}{n} \leq t_{j n}-t_{j-1, n} \leq \frac{C_{2}}{n}
$$

and hence

$$
C_{3} \leq \rho_{j}-\rho_{j-1} \leq C_{4}
$$

Next, we can deduce:

\section{Lemma 6.4}

Assume (5.10) and let

$$
\Lambda=\sup _{x \in \mathbb{R}} f(x, x) .
$$

For each real $a, f(a, \cdot)$ is entire of exponential type $\sigma=\pi \Lambda$.

\section{Proof}

In view of Lemma 6.1, it suffices to show that $f(0, \cdot)$ is entire of exponential type $\sigma=\Lambda \pi$. To do this, we consider the zero distribution of $f(0, \cdot)$. We again need the Markov-Stieltjes inequalities [4, p. 33] associated with these zeros: for each $1 \leq \ell \leq n$,

$$
\sum_{j=1}^{\ell-1} \lambda_{n}\left(t_{j n}\right) \leq \int_{-\infty}^{t_{\ell n}} d \mu \leq \sum_{j=1}^{\ell} \lambda_{n}\left(t_{j n}\right) .
$$

We consider this also for $k$ :

$$
\sum_{j=1}^{k-1} \lambda_{n}\left(t_{j n}\right) \leq \int_{-\infty}^{t_{k n}} d \mu \leq \sum_{j=1}^{k} \lambda_{n}\left(t_{j n}\right) .
$$


Assume $\ell<k$. Subtracting the relevant parts of the two-sets of inequalities yields

$$
\sum_{j=\ell+1}^{k-1} \lambda_{n}\left(t_{j n}\right) \leq \int_{t_{\ell n}}^{t_{k n}} d \mu(t)
$$

Now assume that $t_{\ell n}, t_{k n} \in J_{2}$. Then by absolute continuity of $\mu$ in $J_{2}$, and the substitution $t=\xi_{n}+\frac{s}{\tilde{K}_{n}\left(\xi_{n}, \xi_{n}\right)}$, we obtain

$$
\sum_{j=\ell+1}^{k-1} \frac{K_{n}\left(\xi_{n}, \xi_{n}\right)}{K_{n}\left(t_{j n}, t_{j n}\right)} \leq \int_{\rho_{\ell n}}^{\rho_{k n}} \frac{w\left(\xi_{n}+\frac{s}{\tilde{K}_{n}\left(\xi_{n}, \xi_{n}\right)}\right)}{w\left(\xi_{n}\right)} d s .
$$

Now let $n \rightarrow \infty$ through $\mathcal{S}$. In view of (6.6), and our hypothesis (5.10), the last right-hand side converges to

$$
\int_{\rho_{\ell}}^{\rho_{k}} d s=\rho_{k}-\rho_{\ell}
$$

Next, for each fixed $j$, as $n \rightarrow \infty$ through $\mathcal{S}$,

$$
\begin{aligned}
\frac{K_{n}\left(t_{j n}, t_{j n}\right)}{K_{n}\left(\xi_{n}, \xi_{n}\right)} & =\frac{K_{n}\left(\xi_{n}+\frac{\rho_{j n}}{\tilde{K}_{n}\left(\xi_{n}, \xi_{n}\right)}, \xi_{n}+\frac{\rho_{j n}}{\tilde{K}_{n}\left(\xi_{n}, \xi_{n}\right)}\right)}{K_{n}\left(\xi_{n}, \xi_{n}\right)}=f_{n}\left(\rho_{j n}, \rho_{j n}\right) \\
& \rightarrow f\left(\rho_{j}, \rho_{j}\right) .
\end{aligned}
$$

Thus for each fixed $k, \ell$,

$$
\sum_{j=\ell+1}^{k-1} \frac{1}{f\left(\rho_{j}, \rho_{j}\right)} \leq \rho_{k}-\rho_{\ell}
$$

As $f\left(\rho_{j}, \rho_{j}\right) \leq \Lambda$ for all $j$, we obtain

$$
k-\ell-1 \leq \Lambda\left(\rho_{k}-\rho_{\ell}\right) .
$$

This also implies $\rho_{\ell+2}-\rho_{\ell} \geq C>0$, so $f(0, \cdot)$ has at most double zeros. Moreover, because $\left\{\rho_{j n}\right\}$ are simple zeros of $f_{n}(0, \cdot), \rho_{k}$ can only be a double zero of $f(0, \cdot)$ if it is repeated in the sequence $\left\{\rho_{j}\right\}$. Then, in the interval $\left[\rho_{\ell}, \rho_{k}\right]$, the total number of zeros of $f(0, \cdot)$, namely $k-\ell+1$ or $k-\ell+2$ or $k-\ell+3$, if 0 does not belong to $[k, \ell]$, and $k-\ell$ or $k-\ell+1$ or $k-\ell+2$ if it does, is at most $\Lambda\left(\rho_{\ell}-\rho_{k}\right)+4$. Let us denote by $n(r)$ the number of zeros of $f(0, \cdot)$ in $[-r, r]$ (or equivalently in the ball centre 0 , radius $r$ ). In view of the fact that $C_{1} \leq \rho_{j+2}-\rho_{j} \leq C_{2}$ and there are infinitely many $\left\{\rho_{j}\right\}$, we can choose $\rho_{k}$ a bounded distance from $r$, and $\rho_{\ell}$ a bounded distance from $-r$. We obtain that $n(r)$ is at most the number of zeros in $\left[\rho_{\ell}, \rho_{k}\right]$ plus $O(1)$, and hence at most $\Lambda\left(\rho_{\ell}-\rho_{k}\right)+O(1)$. So

$$
n(r) \leq 2 \Lambda r+O(1) \text {. }
$$

Then, recalling (4.3),

$$
\frac{\sigma}{\pi}=\lim _{r \rightarrow \infty} \frac{n(r)}{2 r} \leq \Lambda
$$


Together with our lower bound $\sigma \geq \pi \Lambda$ from Lemma 6.2, we obtain the result.

\section{Remark}

Together with with Lemma 6.2, this gives

$$
\begin{aligned}
& \int_{-\infty}^{\infty}\left(\frac{f(a, s)}{f(a, a)}-\frac{\sin \sigma(a-s)}{\sigma(a-s)}\right)^{2} d s \\
\leq & \frac{1}{f(a, a)}-\frac{1}{\sup _{x \in \mathbb{R}} f(x, x)} .
\end{aligned}
$$

In particular, if $a$ attains the sup, so that $f(a, a)=\sup _{x \in \mathbb{R}} f(x, x)$, then for all $s \in \mathbb{R}$,

$$
\frac{f(a, s)}{f(a, a)}=\frac{\sin \sigma(a-s)}{\sigma(a-s)} .
$$

If the sup is not attained at any finite point, then instead we obtain a sequence $\left\{a_{k}\right\}$ with $\lim _{k \rightarrow \infty}\left|a_{k}\right|=\infty$, and

$$
\lim _{k \rightarrow \infty} \int_{-\infty}^{\infty}\left(\frac{f\left(a_{k}, s\right)}{f\left(a_{k}, a_{k}\right)}-\frac{\sin \sigma\left(a_{k}-s\right)}{\sigma\left(a_{k}-s\right)}\right)^{2} d s=0 .
$$

In the converse direction, we note that

$$
\begin{aligned}
& \int_{-\infty}^{\infty}\left(\frac{f(a, s)}{f(a, a)}-\frac{\sin \sigma(a-s)}{\sigma(a-s)}\right)^{2} d s \\
= & \frac{1}{f(a, a)^{2}} \int_{-\infty}^{\infty} f(a, s)^{2} d s-\frac{1}{\sup _{x \in \mathbb{R}} f(x, x)} .
\end{aligned}
$$

Then the desired identity (6.9) is true iff

$$
\int_{-\infty}^{\infty} f(a, s)^{2} d s=f(a, a)^{2} / \sup _{x \in \mathbb{R}} f(x, x) .
$$

Note that (1.6) is not assumed for any of this! In fact up till now we have not used the hypothesis (1.6).

\section{Proof of Theorem 1.2}

Obviously (II) implies (I), so we prove only (I) implies (II). Since $\tilde{K}_{n}\left(\xi_{n}, \xi_{n}\right) \sim$ $n$, our hypothesis (1.6) implies also that

$$
\lim _{n \rightarrow \infty} f_{n}(a, a)=\lim _{n \rightarrow \infty} \frac{K_{n}\left(\xi_{n}+\frac{a}{\tilde{K}_{n}\left(\xi_{n}, \xi_{n}\right)}, \xi_{n}+\frac{a}{\tilde{K}_{n}\left(\xi_{n}, \xi_{n}\right)}\right)}{K_{n}\left(\xi_{n}, \xi_{n}\right)}=1,
$$

and hence

$$
f(a, a)=1 \text { for all real } a .
$$

(It is only here that we need the uniformity of (1.6) in $\xi$ and $a$ ). So

$$
\Lambda=\sup _{x \in \mathbb{R}} f(x, x)=1 .
$$


By Lemma 6.4, for each fixed $a, f(a, \cdot)$ is entire of exponential type $\sigma=\pi$. By (6.3), we then obtain, for each real $a$,

$$
\int_{-\infty}^{\infty}\left(\frac{f(a, s)}{f(a, a)}-\frac{\sin \pi(s-a)}{\pi(s-a)}\right)^{2} d s=0,
$$

that is

$$
f(a, s)=\frac{\sin \pi(s-a)}{\pi(s-a)} .
$$

By convergence continuation,

$$
\lim _{n \rightarrow \infty, n \in \mathcal{S}} \frac{K_{n}\left(\xi_{n}+\frac{a}{\tilde{K}_{n}\left(\xi_{n}, \xi_{n}\right)}, \xi_{n}+\frac{b}{\tilde{K}_{n}\left(\xi_{n}, \xi_{n}\right)}\right)}{K_{n}\left(\xi_{n}, \xi_{n}\right)}=\frac{\sin \pi(a-b)}{\pi(a-b)},
$$

uniformly for $a, b$ in compact subsets of the plane. (Recall that the left-hand side is uniformly bounded for such $a, b)$. As the limit function is independent of the subsequence $\mathcal{S}$, we obtain

$$
\lim _{n \rightarrow \infty} \frac{K_{n}\left(\xi_{n}+\frac{a}{\tilde{K}_{n}\left(\xi_{n}, \xi_{n}\right)}, \xi_{n}+\frac{b}{\tilde{K}_{n}\left(\xi_{n}, \xi_{n}\right)}\right)}{K_{n}\left(\xi_{n}, \xi_{n}\right)}=\frac{\sin \pi(a-b)}{\pi(a-b)},
$$

again with the appropriate uniformity in $a, b$. Finally as $\left\{\xi_{n}\right\}$ can be any sequence in $J$, we obtain the conclusion (1.7) of Theorem 1.2, uniformly for $\xi \in J$.

\section{Proof of Theorem 1.1}

This follows immediately from Theorem 1.2. Indeed, the uniformity of (1.11) for $\xi \in J$ follows easily from the assumed continuity of $w$ (regarded as a function defined on all of $\operatorname{supp}[\mu])$ at each point of compact $J$.

\section{Acknowledgement}

The author thanks Eli Levin for observing that the hypothesis (1.6) in Theorem 1.1 must be assumed to hold uniformly in $\xi$ and $a$. The author also thanks Barry Simon for several corrections and remarks that improved the manuscript. In particular, he observed that (4.3) requires more than just the Cartwright class.

\section{REFERENCES}

[1] J. Baik, T. Kriecherbauer, K. T-R. McLaughlin, P.D. Miller, Uniform Asymptotics for Polynomials Orthogonal with respect to a General Class of Discrete Weights and Universality Results for Associated Ensembles, Princeton Annals of Mathematics Studies, 2006.

[2] P. Deift, Orthogonal Polynomials and Random Matrices: A Riemann-Hilbert Approach, Courant Institute Lecture Notes, Vol. 3, New York University Pres, New York, 1999.

[3] M. Findley, Universality for Regular Measures satisfying Szego's Condition, manuscript.

[4] G. Freud, Orthogonal Polynomials, Pergamon Press/ Akademiai Kiado, Budapest, 1971. 
[5] I.S. Gradshteyn and I.M. Ryzhik, Table of Integrals, Series and Products, Academic Press, San Diego, 1980.

[6] J.R. Higgins, Sampling Theory in Fourier and Signal Analysis: Foundations, Oxford University Press, Oxford, 1996.

[7] P. Koosis, The Logarithmic Integral I, Cambridge University Press, Cambridge, 1988.

[8] A.B. Kuijlaars and M. Vanlessen, Universality for Eigenvalue Correlations from the Modified Jacobi Unitary Ensemble, International Maths. Research Notices, 30(2002), 1575-1600.

[9] Y. Last and B. Simon, Fine Structure of the Zeros of Orthogonal Polynomials IV. A Priori Bounds and Clock Behavior, to appear in Communications in Pure and Applied Mathematics.

[10] B. Ya. Levin, in collaboration with Yu. Lyubarskii, M. Sodin, V. Tkachenko, Lectures on Entire Functions, Translations of Mathematical Monographs, Vol. 150, American Mathematical Society, Providence, 1996.

[11] Eli Levin and D.S. Lubinsky, Universality Limits for Exponential Weights, to appear in Constructive Approximation.

[12] Eli Levin and D.S. Lubinsky, Applications of Universality Limits to Zeros and Reproducing Kernels of Orthogonal Polynomials, to appear in Journal of Approximation Theory.

[13] Eli Levin and D.S. Lubinsky, Universality Limits Involving Orthogonal Polynomials on the Unit Circle, to appear in Computational Methods and Function Theory.

[14] Eli Levin and D.S. Lubinsky, Universality Limits in the Bulk for Varying Measures, manuscript.

[15] D.S. Lubinsky, A New Approach to Universality Limits involving Orthogonal Polynomials, to appear in Annals of Mathematics.

[16] D.S. Lubinsky, A New Approach to Universality Limits at the Edge of the Spectrum, to appear in Contemporary Mathematics.

[17] D.S. Lubinsky, Mutually Regular Measures have Similar Universality Limits, to appear in Proceedings of Twelfth Texas Conference on Approximation Theory.

[18] M.L. Mehta, Random Matrices, 2nd edn., Academic Press, Boston, 1991.

[19] P. Nevai, Orthogonal Polynomials, Memoirs of the AMS no. 213 (1979).

[20] P. Nevai, Geza Freud, Orthogonal Polynomials and Christoffel Functions: A Case Study, J. Approx. Theory, 48(1986), 3-167.

[21] B. Simon, Orthogonal Polynomials on the Unit Circle, Parts 1 and 2, American Mathematical Society, Providence, 2005.

[22] B. Simon, Two Extensions of Lubinsky's Universality Theorem, to appear in Journal d'Analyse de Mathematique.

[23] H. Stahl and V. Totik, General Orthogonal Polynomials, Cambridge University Press, Cambridge, 1992.

[24] F. Stenger, Numerical Methods Based on Sinc and Analytic Functions, Springer, New York, 1993.

[25] V. Totik, Asymptotics for Christoffel Functions for General Measures on the Real Line, J. d'Analyse Math., 81(2000), 283-303.

[26] V. Totik, Universality and fine zero spacing on general sets, manuscript.

School of Mathematics, Georgia Institute of Technology, Atlanta, GA 30332-0160, USA., LUBINSKY@MATH.GATECH.EDU 